\title{
Preparation of $\mathrm{SnO}_{2} / \mathrm{C}$ biomorphic materials by biotemplating from ramie fibres
}

\author{
XIN-HAI HE ${ }^{\mathrm{a}, \mathrm{b}}$, LE-HUA QI ${ }^{\mathrm{a}, *}, J$ JUN-BO WANG ${ }^{\mathrm{b}}$, MING-QIAN SHEN ${ }^{\mathrm{b}}$, WEI CHANG ${ }^{\mathrm{b}}$, \\ CHONG FU ${ }^{\mathrm{b}}$, MIN-GE YANG ${ }^{\mathrm{b}}$ and XIAO-LEI SU ${ }^{\mathrm{b}}$ \\ ${ }^{a}$ School of Mechatronics, Northwestern Polytechnical University, Xi'an 710072, P.R.China \\ ${ }^{\mathrm{b}}$ College of Mechanical \& Electrical Engineering, Xi'an Polytechnic University, Xi'an 710048, P.R.China
}

MS received 15 October 2010; revised 31 December 2010

\begin{abstract}
A new $\mathrm{SnO}_{2} / \mathrm{C}$ biomorphic material has been prepared by molding into a composite billet and carbothermal-reduction under vacuum from ramie fibres/Sn $(\mathrm{OH})_{4}$ precursors. The phase composition and microstructure of the prepared materials were characterized. The effects of the carbonization temperature, holding time and other factors on the crystal structure, morphology and ingredients of the prepared samples were discussed. The results showed that the sintering temperature and holding time have significant effects on the final products. When the sintering temperature reached $480^{\circ} \mathrm{C}$, the $\mathrm{SnO}_{2} / \mathrm{C}$ biomorphic materials were synthesized successfully.
\end{abstract}

Keywords. Biomorphic materials; carbothermal-reduction; biotemplate.

\section{Introduction}

Biomorphic materials, as a new kind of porous carbon materials, are usually fabricated by carbonizing wood or woody materials impregnated with phenolic resin under vacuum at an elevated temperature of $300 \sim 2800^{\circ} \mathrm{C}$. By selective artificial compounding, biomorphic materials not only maintain the micro-fine structure of the natural biological materials, but also is endowed with new features and functions. This kind of materials is of great application potential in many industrial areas such as absorbents, sensors, catalyst carrier materials, self-lubricating materials, biomedical materials, heat insulating materials and electromagnetic shielding materials (Griel 2001; Zhang et al 2004; Odeshi et al 2006), etc. In recent years, a variety of biological materials have been taken as bio-templates to prepare biomorphic materials, such as wood (Min et al 2006; Ozao et al 2006; Kaul and Faber 2008), bamboo (Dong 2009), paper (Yang et al 2008), cotton (Amirthan et al 2009), etc. And various oxides (Dong et al 2007), carbide (Sun et al 2004; Kim et al 2006; Martinez-Escandell et al 2009) and nitride (Min et al 2008; Rambo et al 2008) biomorphic materials have been prepared from biotemplates by sol-gel and carbothermal reduction method (Qian and Jin 2006), molten metal infiltration (Wang et al 2006a, b), reactive infiltration of liquid Si (Mallick et al 2007), etc. Furthermore, the researchers have explored deeply into the defects of biomorphic materials, such as poor homogeneity of structure, low mechanical properties,

\footnotetext{
*Author for correspondence (qilehua@nwpu.edu.cn)
}

cracking, etc which lays the theoretical and experimental basis for application of the materials.

Ramie is a perennial herbaceous plant, which can be annually harvested in a great amount 2 to 3 times, so the yield of its natural fibres is very rich. As the prepared template for biomorphic materials, ramie fibres have good uniformity of impregnation and controllable texture compared with wood, bamboo and some other template materials.

In the present work, $\mathrm{SnO}_{2} / \mathrm{C}$ biomorphic materials have been fabricated by carbothermal-reduction under vacuum from ramie fibres biotemplates which has already impregnated with $\mathrm{Sn}(\mathrm{OH})_{4}$ sol using ultrasonic technique. The phase composition and microstructure of the materials are analysed and characterized. The mechanism of preparation has been discussed.

\section{Experimental}

\subsection{Materials preparation}

The ramie fibres, crystalline tin tetrachloride $\left(\mathrm{SnCl}_{4} \cdot 5 \mathrm{H}_{2} \mathrm{O}\right.$ AR), polyethylene glycol (PEG AR), ammonia $\left(\mathrm{NH}_{3} \cdot \mathrm{H}_{2} \mathrm{O}\right.$ $\mathrm{AR}$ ) and deionized water were used as raw materials. Firstly, the PEG $(5 \mathrm{~g} / \mathrm{L})$ was added to $0.05 \mathrm{~mol} / \mathrm{L} \mathrm{SnCl}_{4}$ solution which was prepared by dissolving crystalline $\mathrm{SnCl}_{4} \cdot 5 \mathrm{H}_{2} \mathrm{O}$ with deionized water. Secondly, when the solution was stirred until it was clear and transparent, and then $\mathrm{NH}_{3} \cdot \mathrm{H}_{2} \mathrm{O}$ was added to the $\mathrm{SnCl}_{4}$ solution slowly, stirring was continued till $\mathrm{pH}$ value reached $2-2 \cdot 5$. Then, it was washed by deionized water until no chloride ion was found in the precipitation. At last, $6.5 \mathrm{wt} \%$ hydrosol of $\mathrm{Sn}(\mathrm{OH})_{4}$ was prepared. 
To improve the impregnation ratio, ultrasonic vibration technology was used to impregnate ramie fibres with $\mathrm{Sn}(\mathrm{OH})_{4}$ hydrosol which was prepared earlier and the treatment process of impregnation-drying was repeated several times. Ultimately the ramie fibres/Sn $(\mathrm{OH})_{4}$ precursor was obtained after drying it in an oven at $80^{\circ} \mathrm{C}$ for $24 \mathrm{~h}$, and sintering it in a vacuum hot pressing furnace to $480^{\circ} \mathrm{C}, 560^{\circ} \mathrm{C}$ and $630^{\circ} \mathrm{C}$ for $20-60 \mathrm{~min}$ in the graphite crucibles. Finally, the samples of $\mathrm{SnO}_{2} / \mathrm{C}$ biomorphic materials were prepared after annealing at $150^{\circ} \mathrm{C}$ for $2.5 \mathrm{~h}$. Fabrication scheme of $\mathrm{SnO}_{2} / \mathrm{C}$ biomorphic materials is shown in figure 1. Samples used in the experiment in different sintering conditions are shown in table 1.

\subsection{Characterization}

The differential scanning calorimetry and thermogravimetry (DSC/TG: TGA/SDTA851e, METTLER TOLEDO, Switzerland) were used to analyse and characterize the

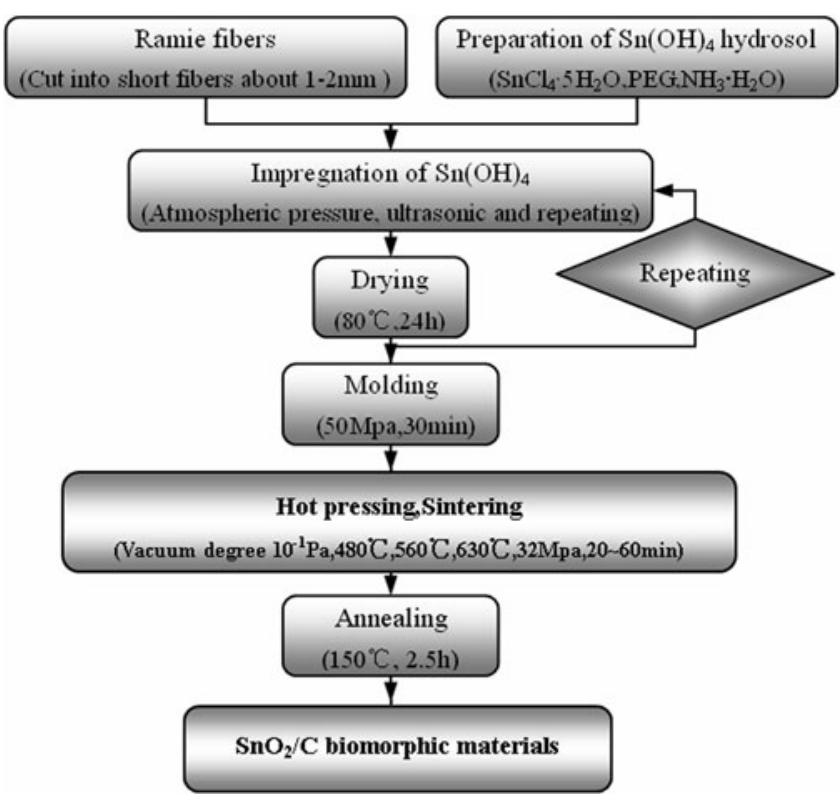

Figure 1. Fabrication scheme of $\mathrm{SnO}_{2} / \mathrm{C}$ biomorphic materials.

Table 1. Samples used in experiment in different sintering conditions (weight ratio of ramie/ $\mathrm{Sn}(\mathrm{OH})_{4} \approx 1 / 1 \cdot 3$ ).

\begin{tabular}{lccc}
\hline Samples & $\begin{array}{c}\text { Temperature } \\
\left({ }^{\circ} \mathrm{C}\right)\end{array}$ & $\begin{array}{c}\text { Heating rate } \\
\left({ }^{\circ} \mathrm{C} / \mathrm{min}\right)\end{array}$ & $\begin{array}{c}\text { Holding time } \\
(\mathrm{min})\end{array}$ \\
\hline $1^{\#}$ & 560 & 5 & 20 \\
$2^{\#}$ & 560 & 5 & 40 \\
$3^{\#}$ & 560 & 5 & 60 \\
$4^{\#}$ & 480 & 5 & 40 \\
$5^{\#}$ & 630 & 5 & 40 \\
$6^{\#}$ & 630 & 8 & 60 \\
\hline
\end{tabular}

pyrolytic behaviour of ramie fibres, $\mathrm{Sn}(\mathrm{OH})_{4}$ powder and the ramie fibres/ $\mathrm{Sn}(\mathrm{OH})_{4}$ precursor. For crystalline phase identification, X-ray diffraction was measured on a X-ray diffractometer (MAXima_X XRD-7000, Shimadzu, Japan) using $\mathrm{Cu}$ radiation produced at $40 \mathrm{kV}$ and $40 \mathrm{~mA}$, at $0.02^{\circ} \mathrm{C}$ step and at a scanning rate of $10^{\circ} \mathrm{C} / \mathrm{min}$. The microstructure morphology was observed with a scanning electron microscope (SEM: JSM-6700F, JEOL, Japan) and elemental analysis was also carried out at several points of the observed area by using energy dispersive spectroscopy (EDS). FTIR measurements were carried out in the wavenumber range between 4000 and $500 \mathrm{~cm}^{-1}$ by a NICOLET 5700 (Thermo, USA) spectrophotometer.

\section{Results and discussion}

\subsection{DSC-TG analysis}

DSC-TG curves of $\mathrm{Sn}(\mathrm{OH})_{4}$ powder (shown in figure 2) reveals the pyrolytic behaviour of $\mathrm{Sn}(\mathrm{OH})_{4}$. It shows that there is a endothermic valley at $70^{\circ} \mathrm{C}$ because the $\mathrm{Sn}(\mathrm{OH})_{4}$ powder is not completely dry and releases some water. The exothermic peak at $345.7^{\circ} \mathrm{C}$ is related to the heat effect of decomposition of $\mathrm{Sn}(\mathrm{OH})_{4}$ into $\mathrm{SnO}_{2}$ (Junbo et al 2006). It can be assumed that the reaction mainly occurs at about $345.7^{\circ} \mathrm{C}$. The reaction involved in this step is

$$
\mathrm{Sn}(\mathrm{OH})_{4}(s) \stackrel{345 \cdot 7^{\circ} \mathrm{C}}{\longrightarrow} \mathrm{SnO}_{2}(s)+2 \mathrm{H}_{2} \mathrm{O}(g) .
$$

Comparing the different thermal weight losses of ramie fibres/Sn $(\mathrm{OH})_{4}$ precursors at two kinds of heating rate (shown in figure 3), it can be found that the heating rate of $5^{\circ} \mathrm{C} / \mathrm{min}$, pyrolysis starts at $189^{\circ} \mathrm{C}$ and ends at $363^{\circ} \mathrm{C}$. At the heating rate of $8^{\circ} \mathrm{C} / \mathrm{min}$ pyrolysis starts at $217^{\circ} \mathrm{C}$ and ends at $422^{\circ} \mathrm{C}$. It can be concluded that thermal decomposition curve shifts to the right, and steepens at high heating rate, which is attributed to fast decomposition but lagging heat conduction.

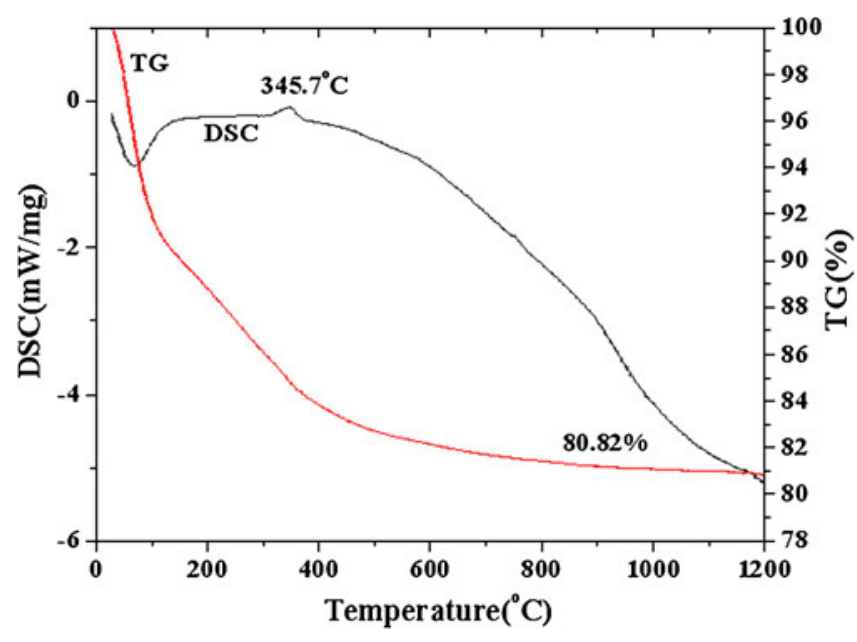

Figure 2. DSC-TG curves of $\mathrm{Sn}(\mathrm{OH})_{4}$ 
As a result, pyrolytic reactions lag behind. In the preparation of biomorphic materials process, it is found that the sample has obvious cracks and deformation at high heating rate. It is mainly due to the uneven heat conduction in the sample which results in a large temperature gradient. Therefore, the appropriate heating rate is $5^{\circ} \mathrm{C} / \mathrm{min}$.

\subsection{XRD analysis}

From XRD patterns of samples at different sintering temperatures (shown in figure 4), it can be seen that the diffraction peaks of carbon derived from ramie fibres are broad dispersion peaks when the sintering temperature is $480^{\circ} \mathrm{C}$ and $560^{\circ} \mathrm{C}$. Even if the sintering temperature is $630^{\circ} \mathrm{C}$, there is no significant change with the peaks. It indicates that the ramie carbon matrix of $\mathrm{SnO}_{2} / \mathrm{C}$ biomorphic materials is a typical amorphous carbon, which is a turbostratic carbon

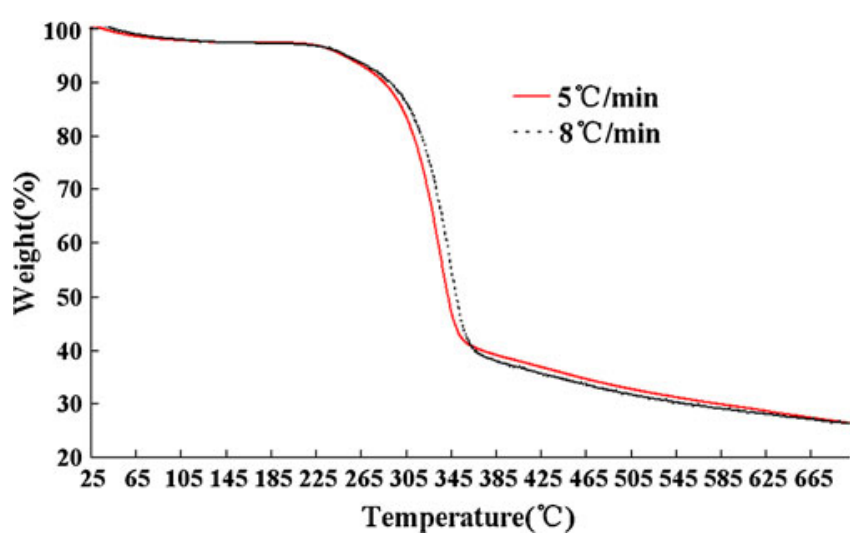

Figure 3. Different TG of two models of heating to precursors.

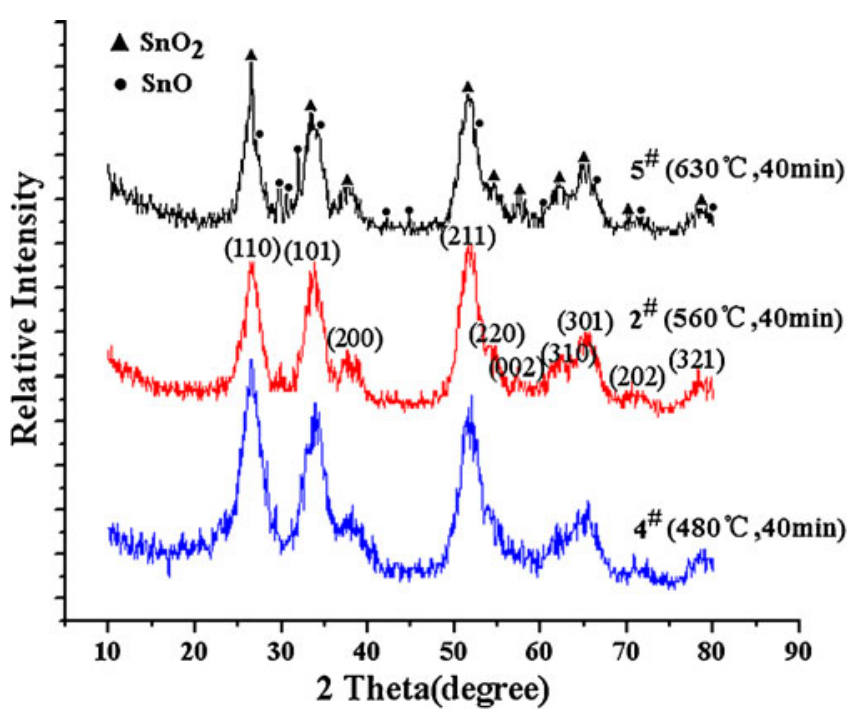

Figure 4. XRD patterns of samples prepared at different sintering temperatures (holding time, $40 \mathrm{~min}$ ). material composed of microcrystalline graphite. $\mathrm{SnO}_{2}$ is produced by the pyrolysis of $\mathrm{Sn}(\mathrm{OH})_{4}$ at $345^{\circ} \mathrm{C}$, and there is no significant change in the intensity of diffraction peaks of $\mathrm{SnO}_{2}$ (JCPDS card No. 41-1445) with increasing carbonization temperature. But when the temperature reaches $630^{\circ} \mathrm{C}$ the apparent diffraction peaks of SnO (JCPDS card No. 06-0395) appear, which indicates that the $\mathrm{SnO}$ has generated.

Through thermodynamic calculation and analysis, under the standard state, when the ambient temperature is above $631^{\circ} \mathrm{C}$ and $689.5^{\circ} \mathrm{C}$, the reduction reactions of $\mathrm{SnO}_{2}$ with $\mathrm{C}$ (2) and (3) will convert into spontaneous reactions from non-spontaneous ones (Ye 2002).

$$
\begin{aligned}
& \mathrm{SnO}_{2}(s)+\mathrm{C}(s) \rightarrow \mathrm{Sn}(s, l)+\mathrm{CO}_{2}(g), \\
& \mathrm{SnO}_{2}(s)+\mathrm{C}(s) \rightarrow \mathrm{SnO}(s)+\mathrm{CO}(g) .
\end{aligned}
$$

Figure 5 shows XRD patterns of samples prepared at different holding times sintered at $560^{\circ} \mathrm{C}$. It can be shown that the (110), (101) and (200) diffraction peaks of $\mathrm{SnO}_{2}$ become sharp with the increase of holding time. The diffraction peaks of SnO and Sn(JCPDS card No. 04-0673) appear when the holding time reaches $60 \mathrm{~min}$. This can be explained due to the fact that $\mathrm{CO}$ and $\mathrm{CO}_{2}$ gases produced by reactions (2) and (3) are extracted timely under vacuum, in which way the occurrence of the reactions (2) and (3) below $630^{\circ} \mathrm{C}$ are promoted, and meanwhile the temperature gradient existing in the samples can also cause localized high temperatures, under which $\mathrm{SnO}_{2}$ is reduced and decomposed into $\mathrm{SnO}$ and $\mathrm{Sn}$ by the effect of strong reducing agent carbon. Moreover, the carbon derived from ramie fibres can also be further carbonized with the extension of holding time (Okabe and Saito 1992).

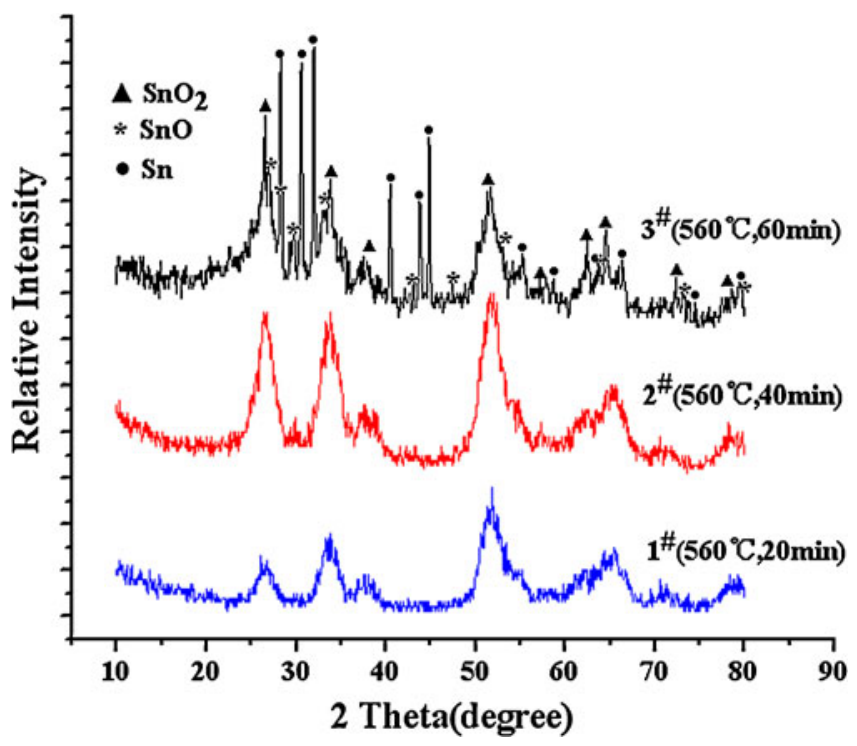

Figure 5. XRD patterns of samples prepared at different holding times (sintering at $560^{\circ} \mathrm{C}$ ). 

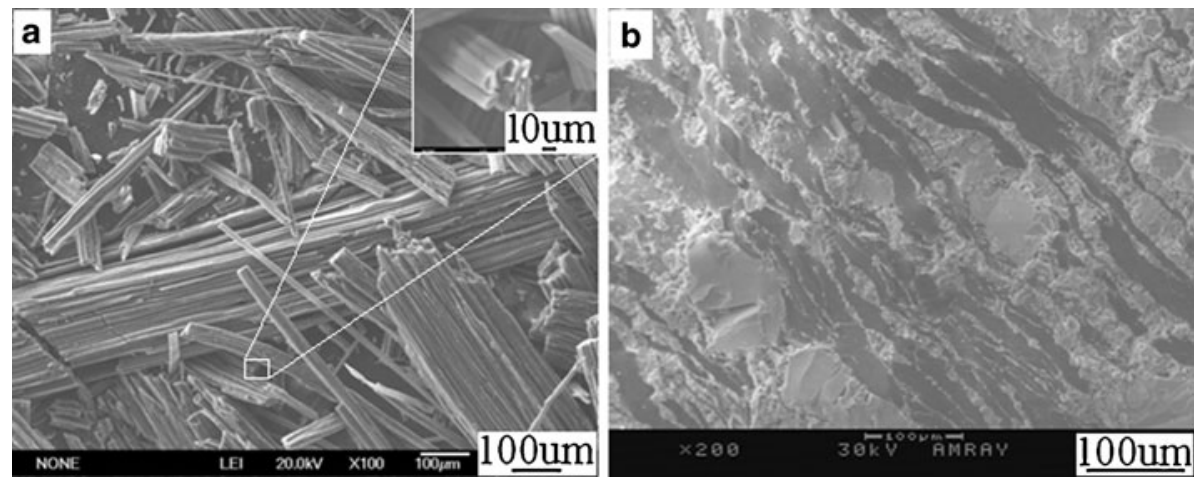

Figure 6. SEM images of carbon biotemplate derived from ramie fibres (a) and carbonized samples (b).
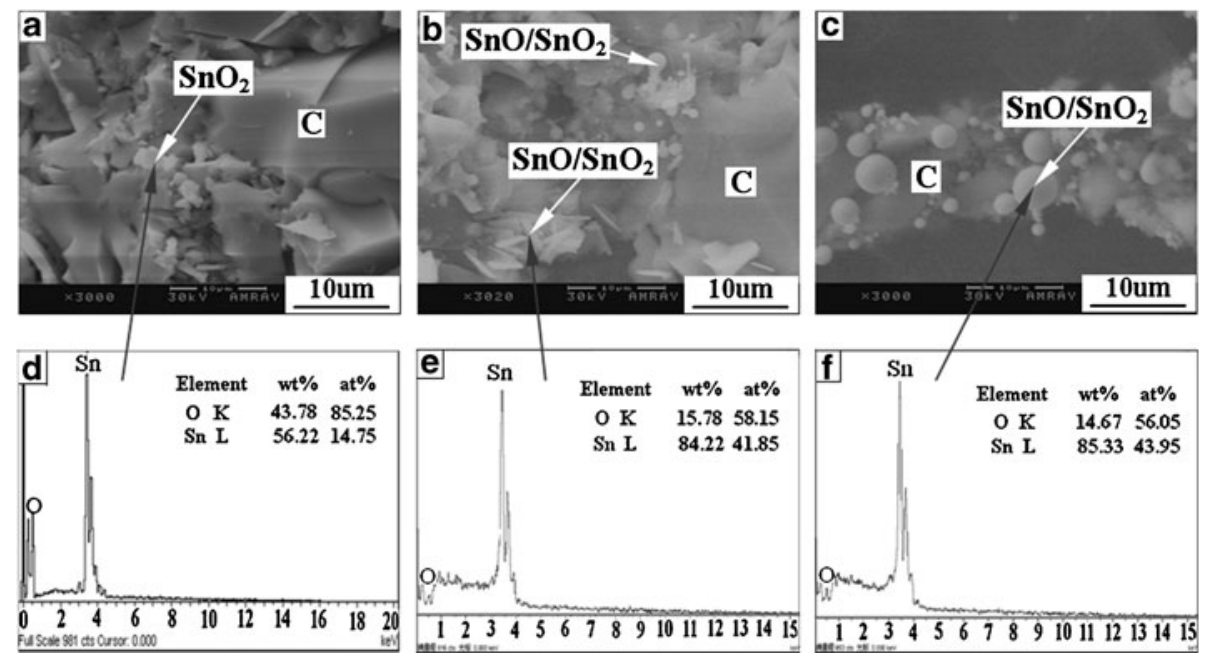

Figure 7. SEM images and corresponding EDS analysis of carbonized samples. SEM images of (a) $4^{\#}$ sample, (b) $3^{\#}$ sample, (c) $5^{\#}$ sample, and EDS analysis of (d) $4^{\#}$ sample, (e) $3^{\#}$ sample and (f) $5^{\#}$ sample.

\subsection{SEM analysis}

From SEM photographs of carbon biotemplate derived from ramie fibres (shown in figure 6(a)), it can be seen that carbon template derived from ramie fibres has a porous structure, the carbon fibres inherit and maintain the natural tracheid structure of ramie fibres, and the orientation of the carbon fibres is random in the template. In the carbon template, in addition to the pores among the carbon fibres, there are about $\Phi 10 \mu \mathrm{m} \sim \Phi 20 \mu \mathrm{m}$ sized circle-like pores in the tracheid of ramie fibres. This structure is conducive to the sol impregnation and the uniform distribution of $\mathrm{SnO}_{2}$ produced by reactions in the carbon matrix.

Through the microstructure of the specimen (shown in figure 6(b)), it can be seen that $\mathrm{SnO}_{2} / \mathrm{C}$ biomorphic materials maintain the structure of carbon template derived from ramie fibres, and $\mathrm{SnO}_{2}$ distributes in the gap of ramie carbon tracheid. However, further studies under high power (3000-fold) SEM photograph find that the porous structure of $\mathrm{SnO}_{2} / \mathrm{C}$ biomorphic materials is not perfectly consistent with the tracheid structure of ramie fibres, because the ramie carbon tracheid deforms under the thermal pressure resulting from the thermal shrinkage of ramie fibres.

Figure 7 shows SEM images and the corresponding EDS analysis of samples carbonized at $480^{\circ} \mathrm{C}, 560^{\circ} \mathrm{C}$ and $630^{\circ} \mathrm{C}$. From the SEM image shown in figure 7(a), it is observed that $\mathrm{SnO}_{2}$ appears as an irregular agglomerate distributing in the tracheid gap of carbon derived from ramie fibres at a sintering temperature of $480^{\circ} \mathrm{C}$. With the increase of sintering temperature and the extension of holding time, the pellet-like components with a diameter of $1 \mu \mathrm{m} \sim 3 \mu \mathrm{m}$ and the tile sheet shaped components with a size of $3 \mu \mathrm{m} \sim 5 \mu \mathrm{m}$ appear in the tracheid gap of carbon derived from ramie fibres (shown in figure $7(\mathrm{~b})$ ). When the sintering temperature reached $630^{\circ} \mathrm{C}$, the components are mainly pellet-like with a diameter of $1 \mu \mathrm{m} \sim 5 \mu \mathrm{m}$ (shown in figure 7(c)). 
According to EDS analysis of the proportion of $\mathrm{Sn}$ and $\mathrm{O}$ (shown in figures 7(d), (e) and (f)), in combination with Sn-O phase diagram (Li 2002) and the XRD diffraction patterns analysis (shown in figures 4 and 5), it can be ascertained that the irregular agglomerate obtained at $480^{\circ} \mathrm{C}$ is primarily $\mathrm{SnO}_{2}$, the tile sheet shaped components are mainly the mixture of $\mathrm{SnO}_{2}$ and $\mathrm{SnO}$ and the weight percentage of the two ingredients are $40.9 \%$ and $59.1 \%$, and the pellet-like components also contain $\mathrm{SnO}_{2}$ and $\mathrm{SnO}$ but their percentages in weight are $29.1 \%$ and $70.9 \%$. The results suggest that, in the starting phase in the sintering process, the $\mathrm{SnO}_{2}$ pyrolysed by $\mathrm{Sn}(\mathrm{OH})_{4}$ is shaped as irregular agglomerate. But due to the action of strong reducing agent carbon, $\mathrm{SnO}_{2}$ changes into the pellet-like or tile sheet shaped mixture of $\mathrm{SnO}_{2}$ and $\mathrm{SnO}$ with increase of sintering temperature and extension of holding time. And the microstructure of the mixture changes from the tile sheet to the pellet-like with the increase of Sn content.

\subsection{FTIR analysis}

In order to confirm and compare the analysis results of $\mathrm{XRD}$ and SEM testing of all the samples, the $\mathrm{SnO}_{2}$ powder obtained from ramie fibres/Sn $(\mathrm{OH})_{4}$ precursors with aerobic sintering at $700^{\circ} \mathrm{C}$ for $40 \mathrm{~min}$ has been prepared. Figure 8 shows FTIR infrared spectra of samples. It is obvious that samples $1^{\#}-5^{\#}$ have similar spectrogram distribution at $4000-750 \mathrm{~cm}^{-1}$. Except in curve $3^{\#}$, the characteristic absorption peaks of $\mathrm{Sn}-\mathrm{O}-\mathrm{Sn}$ are observed at $750-500 \mathrm{~cm}^{-1}$, which is typical of $\mathrm{Sn}-\mathrm{O}$ asymmetric vibration and symmetric vibration peaks.

From the comparison of the samples sintered at $560^{\circ} \mathrm{C}$, it can be found that the characteristic absorption peaks of Sn-O-Sn show width and red-shift with the extension of

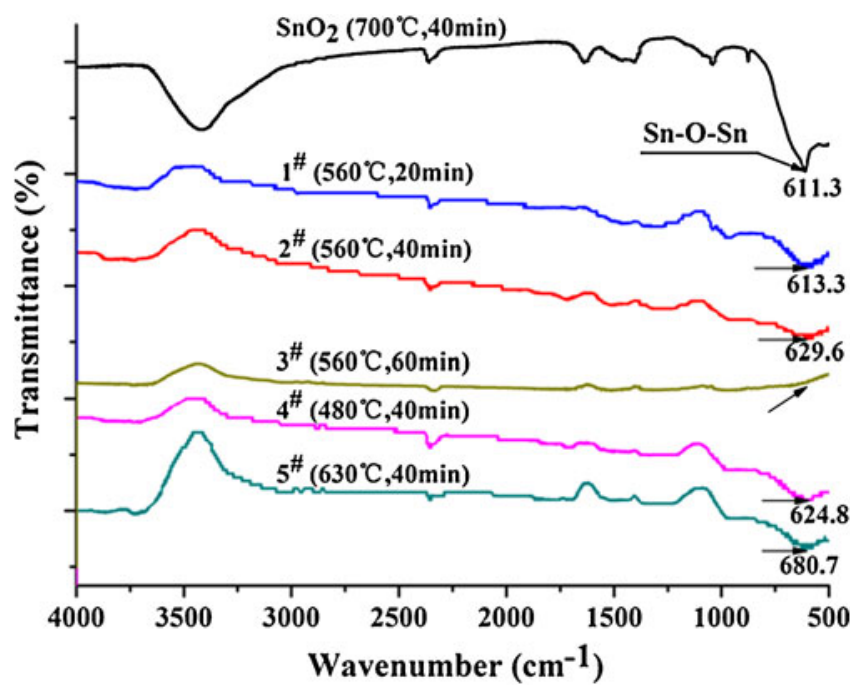

Figure 8. FTIR spectral analysis of samples. holding time. The same results have been found in the prepared samples at the same holding time under different temperatures. However, the red-shift and broadening of peak is closely related to the crystal structures (Wu et al 1996). Therefore, the red-shift of peak of Sn-O-Sn bond is due to the fact that the force generated between ramie carbon and $\mathrm{SnO}_{2}$ peaks result in the $\mathrm{Sn}-\mathrm{O}$ bond. In addition, because the $\mathrm{SnO}$ has been generated, the length of $\mathrm{Sn}-\mathrm{O}$ bond shows greater distribution, which leads to the broadening of absorption peak of Sn-O-Sn bond. The results are consistent with the analysis of XRD and SEM.

\section{Conclusions}

$\mathrm{SnO}_{2} / \mathrm{C}$ biomorphic materials were fabricated by carbothermal-reduction under vacuum from ramie fibres biotemplates which were already been impregnated with $\mathrm{Sn}(\mathrm{OH})_{4}$ sol using ultrasonic technique. The thermal decomposition reaction of ramie fibres mainly concentrate at about $224.7^{\circ} \mathrm{C}$ and $300 \cdot 2^{\circ} \mathrm{C}$. The decomposition of $\mathrm{Sn}(\mathrm{OH})_{4}$ into $\mathrm{SnO}_{2}$ occurs at about $345 \cdot 7^{\circ} \mathrm{C}$. Sintering temperature and holding time have great impact on the final products. The prepared products at the sintering temperature of $480^{\circ} \mathrm{C}$ are $\mathrm{SnO}_{2} / \mathrm{C}$ biomorphic materials, and the prepared products at the sintering temperature of $560^{\circ} \mathrm{C}$ and $630^{\circ} \mathrm{C}$ are $\mathrm{SnO} / \mathrm{SnO}_{2} / \mathrm{C}$ multi-phase ceramic materials. The micro-morphology of $\mathrm{SnO} / \mathrm{SnO}_{2}$ mixture is related to the proportion of $\mathrm{SnO}$ and $\mathrm{SnO}_{2}$, and it changes from the tile sheet to the pellet-like with the increase of Sn content.

However, the preparation of $\mathrm{SnO}_{2} / \mathrm{C}$ biomorphic materials study is limited to the vacuum environment, the research work is not still perfect and the reaction mechanism is not very clear. Therefore, the optimum processing parameters of the preparation of $\mathrm{SnO}_{2} / \mathrm{C}$ biomorphic materials, such as atmosphere, holding time and temperature etc and the reaction mechanism will be studied in future.

\section{Acknowledgements}

The authors wish to express their thanks for the financial support received from the National Natural Science Foundation of China (No. 51002113), Shaanxi Science and Technology Research and Development Program (No. 2011K07-10), the Natural Science Specialized Foundation from Shaanxi Department of Education (No. 09JK449, 2010JK579), Basic Research Foundation of Xi' an Polytechnic University (No. XGJ08010) and Guidance Research Program of China Textile Industry Association (No. 2008046).

\section{References}

Amirthan G, Udayakumar A, BhanuPrasad V V and Balasubramanian M 2009 Ceram. Int. 35967 
Da-Lun Ye 2002 Practical handbook of thermodynamic data of inorganic metallurgy (Beijing: Industry Press)

Dong Liu 2009 Preparation and charactization of bamboo based SiC/C composite biological Mimesis Ceramics, M.Sc. thesis, Beijing Forestry University, Beijing

Dong Qun, Su Huilan, Xu Jiaqiang, Zhang Di and Wang Ruibing 2007 Mater. Lett. 612714

Griel P 2001 J. Eur. Ceram. Soc. 21105

Junbo Wang, Yingmin Li and Minge Yang 2006 Mater. Sci. Eng. B131 230

Kaul V S and Faber K T 2008 Scr. Mater. 58886

Kim Jae-Won, Myoung Sang-Won, Kim Hyeon-Cheol, Lee Je-Hyun, Jung Yeon-Gil and Jo Chang-Yong 2006 Sci. Eng. A434 171

Mallick D, Chakrabarti O P, Majumdar R and Maiti H S 2007 Ceram. Int. 33217

Martinez-Escandell M, Narciso J and Rodriguez-Reinoso F 2009 Carbon 4002

Min Luo, Jiqiang Gao and Xiao Zhang 2006 Rare Metal Mater. Eng. 35133

Min Luo, Jia Cheng and Jing Ma 2008 J. Inorg. Mater. 23763
Odeshi A G, Mucha H and Wielage B 2006 Carbon 441994

Okabe T and Saito K 1992 Meta. Tech. 6234

Ozao Riko, Nishimoto Yuko and Weiping Pan 2006 Therm. Acta 44075

Qian Jun-Min and Jin Zhi-Hao 2006 J. Eur. Ceram. Soc. 261311

Rambo C R, Sieber H and Genova L A 2008 J. Porous Mater. 15 419

Shang Chien Li 2002 Electro-optical properties of Sb and Ta doped $\mathrm{SnO}_{2}$ thin films derived from an ultrasonic atomization process, M.Sc. thesis, National Sun Yat-sen, TaiWan

Sun Binghe, Fan Tongxiang and Zhang Di 2004 Mater. Lett. 58 798

Wang T C, Fan T X, Zhang D and Zhang G D 2006a Mater. Lett. 60 2695

Wang T C, Fan T X, Zhang D and Zhang G D 2006b Carbon 44 900

Xiaohun Wu, Bingsuo Zou, Guilan Zhang, Guoqing Tang, Baolong Yu and Wenju Chen 1996 Chin. J. Semicond. 6416

Yang Gangbin, Liu Yinjuan, Qiao Guanjun, Yang Jianfeng and Wang Hongjie 2008 Mater. Sci. Eng. A492 327

Zhang Di, Sun Binghe and Fan Tongxiang 2004 Sci. China E47 470 